\title{
Mold Mix Antigen IgG Antibody Measurement
}

National Cancer Institute

\section{Source}

National Cancer Institute. Mold Mix Antigen Ig G Antibody Measurement. NCI Thesaurus.

Code C130110.

A measurement of the mold mix antigen IgG antibody in a biological specimen. 